\title{
A Study on Dental Radiography for Peri-Implantitis and Radiation Safety
}

\author{
Neeru Kapur and Meghna Varma \\ Department of Radiology Shree Guru Gobind Singh Tricentary \\ University, Gurugram, Haryana, India \\ Corresponding author email: neeru_fmhs@sgtuniversity.org
}

\begin{abstract}
Dental radiography holds extreme importance in disease diagnosis. The technique has provided several advantages in visualization during complex procedures of dental implantation. The accessibility of the techniques has made the radiologists incautious about the difference between need and ease of practice. The practice of radiography has led to unnecessary exposure to patients due to neglected attitude towards radiation surveillance principles. The preset study evaluates prevalence of different radiography techniques in dental implantation and implant infections. The level of awareness among radiologists has also been studied through the questionnaire based survey. The study has shown that there is need for providing awareness regarding radiation safety of patients and radiation surveillance principles for routine practice of these principles to avoid unnecessary radiation exposure to patients.
\end{abstract}

KEY WORDS: DENTAL RADIOGRAPHY, PERI-APICAL RADIOGRAPHY, PANORAMIC RADIOGRAPHY, PERIIMPLANTITIS, RADIATION SAFETY.

\section{INTRODUCTION}

The practices of imaging in medicine hold importance prior to disease treatments. The efficient imaging technique helps in identifying the disease cause and thus providing most efficient treatments. Disease diagnosis is most essential application of medical imaging as it helps in identification of injuries, bone deformities, structural changes, infections, and teeth root pathologies with many other health issues (V Chauhan et. al. (2019)). In general terms, medical imaging refers to obtaining image so body parts to understand body changes responsible for a disease condition. The imaging techniques help in screening of health conditions before appearance of symptoms. It helps in monitoring diagnosed disease conditions along with studying the effect of treatment against the disease. The field of medical imaging is termed is radiology and doctors specialized in the field are called radiologists. These radiologists or radiology technicians

Biosc Biotech Res Comm P-ISSN: 0974-6455 E-ISSN: 2321-4007

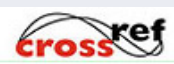

Identifiers and Pagination

Year: 2021 Vol: 14 No (6) Special Issue

Pages: $395-400$

This is an open access article under Creative

Commons License Attribn 4.0 Intl (CC-BY).

DOI: $h$ ttp://dx.doi.org/10.21786/bbrc/14.7.83 or imaging technologists are health professionals trained in specific imaging processes such as specialists of X-rays are radiographers, specialists for ultrasound imaging are sonographers.

The medical imaging techniques are tool to gaining morphological understanding of inter body structures and processes. The basic functioning of an imaging tool depends on three components - sensor, detector and display unit. The sensor obtain a source of energy that can penetrate through the human body, absorbed or diffracted at different angles depending on structures they are directed to, creating signals that are mathematically resolved to obtain image on a display screen(H. Kasban et. al. (2015)). Depending on the type of energy sources there are different techniques that are being used to obtain body images. These energy sources are coupled in the system based on their penetration efficiency to complex body structures (Table 1).

The radiographic techniques become extremely essential when implants are involved. Periapical and panoramic radiographs are mostly used as they are low cost, quick and extremely accessible. Their role in implant dentistry includes surgical planning and post implantitis observations in case of ailments. It helps in finding the locations and orientation of implant to be placed in the dental structures. After placement

\section{Article Information}

Received: 19th May 2021

ccepted after revision: $28^{\text {th }}$ July 2021 
of implant, reproducible and objective measurements are studied for determining implant health status for prevention of failure (J. Prosthet (2008), H. DeBruyn (2013)). Periimplantitis is a condition developed in implants due to bacterial infections in the form of biofilm. The lesions of perimplantitis can be observed by radiography as radiolucent lesions. To obtain accurate results, radiographic film location and light source settings should be optimized with extreme attention to obtain a standard parameter for obtaining alterations free images (B. V. Saberi et. al. (2019)). The main limitation is using periapical radiographs are based on 2-D images which mostly shows inter-proximal area only. The present survey study has been performed to evaluate radiography protocols of dental radiologists during implants analysis along with their awareness about radiation exposure to patients.



Literature Review: The timely diagnosis of periimplantitis can prevent the need for surgery to remove the implants and replace with the fresh one. The surgical methods are mostly used to limit the spread of infection from implant to other regions of the body. Lang et al. suggested cumulative interceptive supportive therapy (CIST) for treatment of peri-implant infection which involved - a) removal debridement deposited on the implant mechanically; b) decontamination by using antiseptics; c) antibiotics treatment to decontaminate the implant; d) surgical procedures to re-establish bone implant which is done when the infection has caused complete loss of osseointegration causing mobility of the implant from its position (N. P. Lang et. al. (2000)). Thus radiography imaging plays important role in identifying the condition of implant infections. In order to solve limitations of 2-D imaging techniques, 3D techniques such as cone beam computed tomography (CBCT) have been developed to obtain improved images of bone height and bone thickness. The technique works in less time with requirement of lower dose (T. Joda et. al. (2015)).

The radiation dose and protocols of imaging have impacted patients' life to a significant extent. The regulation of radiation exposure is necessary to limit the radiation burden on patents. Radiographic imaging techniques 
specifically in dentistry depends on patients' age, oral health and risk factors related to disease symptoms. Dental radiographs have been responsible for $2.5 \%$ of effective radiation doses arising from radiographs and fluoroscopies (American Dental Association et. al. (1987)). Effective radiation doses for dental radiographs are mentioned in Table 2. The number of radiographs required for a particular condition depends on these factors. The practice does not follow 'one size fits all' when duration between subsequent radiography examinations are considered. Several principles have been studied to ensure safety in radiography including ALARA principle which stands for As Low as Reasonably Achievable. The principle is based on the strategy of developing radiation procedures with lowest possible exposure to achieve required image. The principle helps radiologists in taking precautions which helps to ensure the following (T. P. Kumar (2018), https:// blog.universalmedicalinc.com/7-alara-principles-forreducing-radiation-exposure/)

a) Justification of radiation exposure in accordance with its application and benefits

b) Maintenance of radiation levels as low as possible without hampering efficiency of the imaging procedure

c) Maintenance of radiation dose within the permissible limits of exposure to human beings without causing additional health deterioration due to radiations.

Table 2. Questionnaire details used in the study.

\begin{tabular}{|c|c|c|}
\hline Category & Question & Response \\
\hline \multirow{4}{*}{ Demographics } & Age & $<30,31-49,50-64,>65$ \\
\hline & Gender & $\mathrm{M}, \mathrm{F}$ \\
\hline & Working experience & $<4,5-10,11-20,>20$ \\
\hline & $\begin{array}{l}\text { How many dental implants placed per } \\
\text { year? }\end{array}$ & $<30,30-70,>70$ \\
\hline \multirow{5}{*}{ Radiology practices } & $\begin{array}{l}\text { Which radiograph do you prefer for } \\
\text { studying implant location in oral cavity? }\end{array}$ & $\begin{array}{c}\text { Panoramic, Periapical X } \\
\text { ray, CBCT }\end{array}$ \\
\hline & $\begin{array}{l}\text { At what stage do you prefer doing } \\
\text { radiographic imaging? }\end{array}$ & $\begin{array}{l}\text { Immediately post-surgery, } \\
\text { after } 2 \text { weeks, at } \\
\text { uncovering of implant, } \\
\text { during prosthesis delivery } \\
\text { all of the above }\end{array}$ \\
\hline & $\begin{array}{l}\text { Which technique do you use to observe } \\
\text { implant infections such as peri-implantitis? }\end{array}$ & $\begin{array}{l}\text { Panoramic, Periapical } \mathrm{X} \\
\text { ray, } \mathrm{CBCT}\end{array}$ \\
\hline & $\begin{array}{l}\text { Do you think radiology is efficient in } \\
\text { detecting peri-implantitis? }\end{array}$ & Yes, No \\
\hline & $\begin{array}{l}\text { At what stage better imaging of peri- } \\
\text { implantitis is acquired through } \\
\text { radiography? }\end{array}$ & Initial, Moderate, Critical \\
\hline \multirow{5}{*}{$\begin{array}{l}\text { Radiation } \\
\text { awareness }\end{array}$} & $\begin{array}{c}\text { Are you aware of harmful effects of X } \\
\text { rays? }\end{array}$ & Yes, No \\
\hline & Are you aware of ALARA principle? & Yes, No \\
\hline & $\begin{array}{c}\text { Are you aware of radiation protection } \\
\text { protocol? }\end{array}$ & Yes, No \\
\hline & Do you use lead aprons over patients? & Yes, No \\
\hline & $\begin{array}{c}\text { Are you aware of radiation dose } \\
\text { permissible under the exposure limit for } \\
\text { human beings? }\end{array}$ & Yes, No \\
\hline
\end{tabular}


The surveillance of dental radiography is required to maintain a safety standard for patients. There have been very less number of extensive studies that focus on evaluating radiologists practices in dental implant assessment. Clinically, it is evident that there exists lack of evidence in radiology attitude in handling imaging tools for studying implant assessment at post-operative stage. The present study investigates radiology practices and routine habits of dentists while conducting implant post-operative studies. The study also focuses on awareness and knowledge of dental radiologists regarding radiation exposure in accordance with patients' safety.

\section{Research Questions}

1. What is the role of radiology in dentistry and prevalence of different radiological techniques for studying implants infections?

2. What is the level of awareness among radiologists regarding radiation surveillance principles and radiation safety of patients?

\section{MATERIAL AND METHODS}

Survey population: The study utilized anonymous questionnaire based on dental radiographic imaging in implants analysis which was sent through e-mail to dentists practicing radiography in private diagnostic clinics of Uttar Pradesh. The requirements for the survey included list of private diagnostics labs in the state and an active formal email address. Te inclusion criteria of the survey included labs performing at least seven radiology procedures on regular basis. The target population was randomly selected through software to obtain a list of 500 radiologists.

Survey study: The invitation emails had web based questionnaire link which had limitation of to be responded only one by an email address. The questions asked in the survey comprised of three categories of demographics, radiography practices and radiation awareness. Each category comprise of five questions to be responded either by filling the blanks or choosing among the choices. All the questions were close ended except one question which required written input. The survey included a consent form to obtain signed consent for using the data for analysis.

\section{RESULTS AND DISCUSSION}

Importance of different radiographs in dentistry: The results obtained in the survey were statistically analyzed to find the most famous form of radiograph in the domain of dentistry. X ray radiography is mostly used for routine observations as most of the cases entertained by the diagnostics include those of dental carries. According to results obtained in figure $1, \mathrm{X}$ ray radiography is most famous for dental diagnostics in which panoramic and periepial views are equally studied. According to the respondents, panoramic radiographs are mostly used for visualizing misalignment of jaw structures and entire teeth assembly. Periapical radiographs have been prescribed in the case of gum infections or implans alignment. Interestingly, it was found that 2-D visualization technique CBCT has been used by dentists for detailed visualization of tooth structures. Although, CBCT pose problems in terms of contrast and some artefacts during image processing but the technique helps in visualization of overall structure at a better orientation. Overall, it has been found that both $\mathrm{X}$ rays as well as CBCT are quite prevalent in dentistry and $\mathrm{X}$ rays are preferred more by the dentists, which might be due to less awareness of the CBCT and lack of expertise in image processing.

Figure 1: Comparative prevalence of different radiographic techniques in dentistry. CBCT - Cone Beam Computed Tomography

80

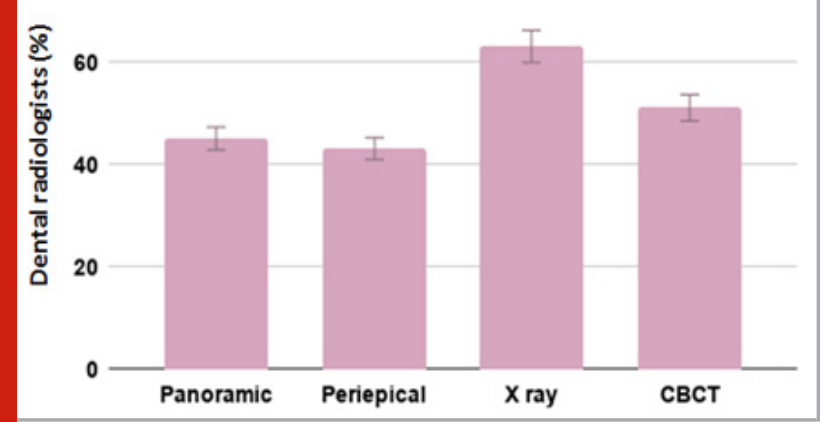

Figure 2: Preference of radiography depending upon dental implantation stage.



Dental radiography for implants: The role of radiographic techniques in implants visualization is extremely essential for proper placement of implant and diagnosis of unconventional changes at proper time to avoid implant failure. The current survey included question regarding the stage at which dental radiography is mostly done. It was designed to understand frequency of radiographs being used for implants. As observed in Figure 2, it has been found that radiography is required mostly during prosthesis delivery within the oral cavity. The result may point to the requirement of visualization to ensure correct alignment of implant with respect to surrounding structures. Other dentists were found to prefer using radiography for visualizing implants during uncovering procedures. Very few people use radiography directly after surgery which may be related with reducing the exposure to the inflamed tissue. Surprisingly, several respondents marked all the stages for their preference of radiography for implants. The result can be attributed to the lack of radiation safety 
awareness among practitioners, due to which reckless use of imaging techniques might be prevailing.

Figure 3: a) Prevalence of different radiographic techniques for peri-implantitis. b) Comparative preference of radiographic according to stages of peri-implantitis.



Dental radiography for implant infections: Periimplantitis is an infection of implants wherein destructive inflammation affects hard and soft tissue surrounding the implants leading to osseointegration with time. Due to this the implant loses its purpose of retention and becomes flexible, loosely attached to the dental plate. To understand the importance of radiography in implantitis, it was asked that what type of radiographs are mostly needed to study infections in implants. As observed in figure 3, it was found that periapical radiographs are mostly used in periimplantitis. The next mostly used radiographs are CBCT. The infections are visualized as radiolucent lesions depending upon the stage of infection. According to respondents, the imaging is usually preferred at moderate and critical stages of implantitis. The initial stages of implantitis may not be resolved properly during radiographic imaging. Moreover limitations of contrast in CBCT may be reason for less preference for peri-implantitis studies.

Radiation awareness among Radiologists: Human body can tolerate certain level of radiations beyond which the radiation exposure can lead to skin diseases and underlying ailments. To study the awareness for radiation exposure to patients, questions were designed regarding understanding and practical utilization of standard radiation procedures. It was asked if radiologists are aware of ALARA principle of radiation safety; it was found that approximately $60 \%$ of dental radiologists have studied ALARA and implement the same in routine diagnosis (Figure 4). Similarly, radiation protocols are followed by less than $50 \%$ of the radiologists, which is to be noted for safety of patients. The specific protocols include protecting patients through lead aprons which is followed by more than $50 \%$ of the patients. Moreover, very less number of radiologists is aware of permissible radiation exposure for humans. This result can be correlated with lack of knowledge about radiation protocols. Overall, the results suggest that there is underestimation of radiation safety measures among radiologists working for dental disease diagnosis. The specific details of radiation protocol including radiograph screen, collimators, radiation dose and importance of avoiding unnecessary radiography should be communicated among radiologists through trainings and survey studies. Further, the radiation dose and number of radiographs based on disease severity could be further explored in future studies.

Figure 4: Awareness of radiation surveillance and radiation safety of patients in dental radiologists.



\section{CONCLUSION}

Radiography is a boon for the medical domain which has elevated the state of disease diagnosis. The technique provides radiographs depicting internal picture of human body. The technique has gained importance in dentistry for visualization of dental structures at both intraoral and extraoral levels. The complexity of dental prosthesis and complication related to the procedures has increased the demand of radiographic techniques. Dentists rely on radiographs of $\mathrm{X}$ ray or $\mathrm{CBCT}$ for determining the health state of an implant in order to prevent implant failure. The major techniques used in implantitis include periapical and panoramic radiography. The present study surveys prevalence of preferred technique to study peri- implantitis. The study provides view of radiographic needs in dentistry along with awareness regarding radiation safety of patients. The study has shown that there is need for providing awareness regarding radiation safety of patients and radiation surveillance principles for routine practice of these principles to avoid unnecessary radiation exposure to patients.

\section{REFERENCES}

Ailuno, G., Iacobazzi, R.M., Lopalco, A., Baldassari, S., Arduino, I., Azzariti, A., Pastorino, S., Caviglioli, G. and Denora, N., 2021. The Pharmaceutical Technology Approach on Imaging Innovations from Italian esearch. Pharmaceutics, 13(8), p.1214.

Broder, J.C., Cameron, S.F., Korn, W.T. and Baccei, S.J., 2018. Creating a radiology quality and safety program: 
principles and pitfalls. Radiographics, 38(6), pp.17861798.

Chauhan, V. and Wilkins, R.C., 2019. A comprehensive review of the literature on the biological effects from dental $\mathrm{X}$-ray exposures. International journal of radiation biology, 95(2), pp.107-119.

De Bruyn, H., Vandeweghe, S., Ruyffelaert, C., Cosyn, J. and Sennerby, L., 2013. Radiographic evaluation of modern oral implants with emphasis on crestal bone level and relevance to peri $\square$ implant health. Periodontology 2000, 62(1), pp.256-270.

Joda, T. and Gallucci, G.O., 2015. The virtual patient in dental medicine. Clinical oral implants research, 26(6), pp.725-726.

Kumar, T.P., Azmi, R., Premkumar, A., Sujatha, S., Devi,
B.Y., Rakesh, N. and Shwetha, V., 2018. Radiation safety protocol in dentistry: A neglected practice. Journal of Orofacial Sciences, 10(1), p.24.

Lang, N.P., Wilson, T.G. and Corbet, E.F., 2000. Biological complications with dental implants: their prevention, diagnosis and treatment Note. Clinical Oral Implants Research: Chapter 9, 11, pp.146-155.Saberi, B.V., Khosravifard, N., Ghandari, F. and Hadinezhad, A., 2019. Detection of peri-implant bone defects using cone-beam computed tomography and digital periapical radiography with parallel and oblique projection. Imaging science in dentistry, 49(4), pp.265-272.

Shetty, A., Almeida, F.T., Ganatra, S., Senior, A. and Pacheco-Pereira, C., 2019. Evidence on radiation dose reduction using rectangular collimation: a systematic review. International dental journal, 69(2), pp.84-97. 